\title{
Primer hallazgo de la familia Amathillopsidae Pirlot, 1934 (Crustacea, Amphipoda, Gammaridea), en el Mar Caribe, con la descripción de una especie nueva
}

\section{First identification of the family Amathillopsidae Pirlot, 1934 (Crustacea, Amphipoda, Gammaridea) in the Caribbean Sea, with the description of a new species}

\section{Carlos Varela ${ }^{1}$}

\section{RESUMEN}

Se describe una especie nueva perteneciente al género Amathillopsis, la cual se caracteriza por carecer de ojos, flagelo accesorio con un solo artejo, telson emarginado distalmente, con un proceso espinoso mediodorsal en los pereionitos 4-7, pleonitos 1-3 y urosomito 1. Esta es la primera cita de una especie de la familia Amathillopsidae Pirlot, 1934 para el Mar Caribe.

Palabras claves: Amphipoda, Gammaridea, Amathillopsidae, Amathillopsis, Mar Caribe.

\begin{abstract}
A new species of the genus Amathillopsis is described. Main characteristics include the absence of eyes, accessory flagellum 1-segmented, distally emarginate telson, medial dorsal spinous process on pereionites 4-7, pleonites 1-3 and urosomite 1 . This is the first record of a species of the family Amathillopsidae Pirlot, 1934 for the Caribbean Sea.
\end{abstract}

Keywords: Amphipoda, Gammaridea, Amathillopsidae, Amathillopsis, Caribbean Sea.

\section{INTRODUCCIÓN}

Los representantes de la familia Amathillopsidae habitan solo las aguas profundas, siendo muy raro su hallazgo. Este grupo ha tenido numerosos cambios con respecto a su actual ubicación taxonómica (Barnard, 1967; Barnard \& Karaman, 1991; Coleman \& Barnard, 1991; Lowry, 2006). Dentro de esta familia, el género Amathillopsis, considerado como cosmopolita (Wakabara \& Serejo, 1999), tiene representantes en todos los océanos. Hasta el presente se han descrito 12 especies en este género, la mayoría de las cuales, cinco especies, $A$. affinis Miers, 1881; A. annectens (Holmes, 1908); A. grevei J. L. Barnard, 1961; A. australis Stebbing, 1883 y A. takahashiae Tomikawa \& Mawatari, 2006, han sido

1 Department of Marine Biology and Ecology, Rosenstiel School of Marine and Atmospheric Science (R.S.M.A.S.), University of Miami. varela06@gmail.com 
registradas en el Océano Pacífico, dos especies, A. septemspinosa Ledoyer, 1978 y A. comorensis Ledoyer, 1986, habitan en el Océano Índico, dos en el Océano Ártico, A. spinigera Heller, 1875 y A. pacifica Gurjanova, 1955, dos en la Antártida, A. charlottae Coleman, 1998 y A. roroi Coleman $\&$ Coleman, 2008 y solo una especie, A. atlantica Chevreux, 1908, ha sido hallada en el Océano Atlántico.

El estudio de material de crustáceos peracáridos marinos depositados en el Museo de Invertebrados Marinos de Rosenstiel School of Marine and Atmospheric Science (R.S.M.A.S.), producto de los viajes de exploración de los B/I Elliot Pillsbury y B/I Gerda, ha permitido encontrar material de especies no descritas para la ciencia y además varios registros de taxones aún no conocidos para el Golfo de México y el Mar Caribe (Varela \& García-Gómez, 2015). En este trabajo se presenta una especie de la familia Amathillopsidae, aún no descrita para la ciencia. La recolecta de dichos ejemplares en el talud frente a Jamaica constituye el primer hallazgo de una especie de la familia Amathillopsidae para el Mar Caribe.

\section{MATERIALES Y MÉTODOS}

El material estudiado fue producto de los viajes de exploración del buque de investigación de la Universidad de Miami, B/I Elliot Pillsbury, en varias localidades del Mar Caribe. Las muestras fueron obtenidas usando una rastra Otter. Los ejemplares de Amathillopsis fueron estudiados bajo el microscopio estereoscópico y las ilustraciones se realizaron con un microscopio biológico con cámara clara acoplada.

Para la identificación del material se ha utilizado a Barnard \& Karaman (1991), Lowry (2006), la tabla comparativa de las especies del género Amathillopsis de Tomikawa \& Mawatari (2006) y el posterior trabajo de Coleman \& Coleman (2008).

El material ha sido depositado en el Museo de Invertebrados Marinos de R.S.M.A.S.

\section{RESULTADOS}

Orden Amphipoda Latreille, 1816 Familia Amathillopsidae Pirlot, 1934 Subfamilia Amathillopsinae Pirlot, 1934 Género Amathillopsis Heller, 1875 Amathillopsis antillensis, sp. nov.

(Figs. 1-5)

Material tipo. Holotipo: Hembra no ovígera. JAMAICA, $17^{\circ} 54^{\prime} \mathrm{N}$, $78^{\circ} 25^{\prime} \mathrm{O}$; recolectada el 7. vii. 1970 , a $1428 \mathrm{~m}$ de profundidad. Depositada en la Colección de Invertebrados Marinos de R.S.M.A.S. UMNL. 32. 10272. Paratipos: Dos hembras no ovígeras, recolectadas en la misma fecha y localidad. Depositadas junto al holotipo UMML. 32. 10271.

Etimología. Esta especie se denomina antillensis por haber sido recolectada en Jamaica, isla perteneciente al grupo de islas caribeñas conocidas como Antillas Mayores.

Diagnosis. Espécimen con flagelo accesorio con solo un artejo; ojo ausente; coxas 1 y 2 con su borde ventral cóncavo; gnatópodo 2 con el lóbulo ventral del carpopodito amplio; coxopoditos 3 y 4 de tamaño normal; incisivo de la 


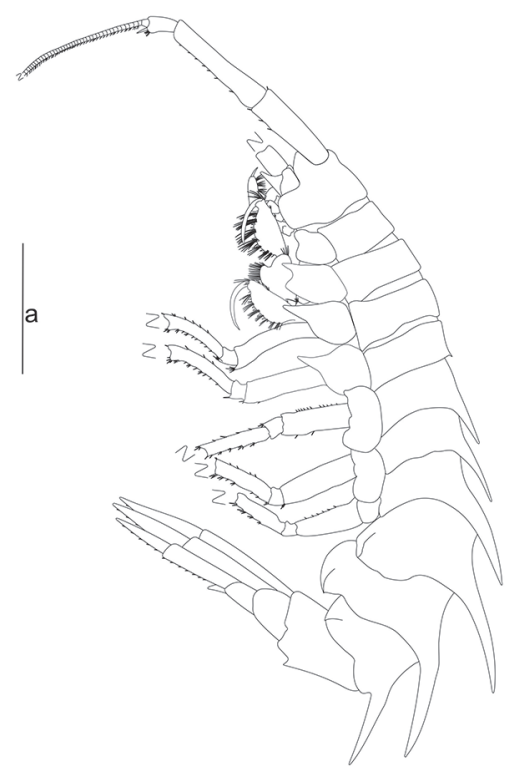

Fig. 1. Amathillopsis antillensis, especie nueva. Vista lateral del holotipo. Escala. a: $5 \mathrm{~mm}$

Fig. 1. Amathillopsis antillensis, new species. Lateral view of the holotype. Scale bar. a: $5 \mathrm{~mm}$

mandíbula izquierda con tres dientes y lacinia mobilis con cinco dientes; relación entre la longitud del artejo 3 del palpo mandibular con respecto al artejo 2 es de 1,5; rama externa del urópodo 3 es más corta que la interna; con proceso espinoso mediodorsal en el urosomito 1 $\mathrm{y}$ telson emarginado distalmente.

Descripción del holotipo. Espécimen de $30.2 \mathrm{~mm}$ de longitud, medido desde el extremo anterior del rostro al extremo distal del telson. Cabeza con rostro corto, ángulo anteroventral ligeramente excavado, lóbulo ocular con un proceso agudo corto. Ojos ausentes. Pereionitos 1-3 subiguales en longitud, sin procesos espinosos dorsales. Pereionito 4 con

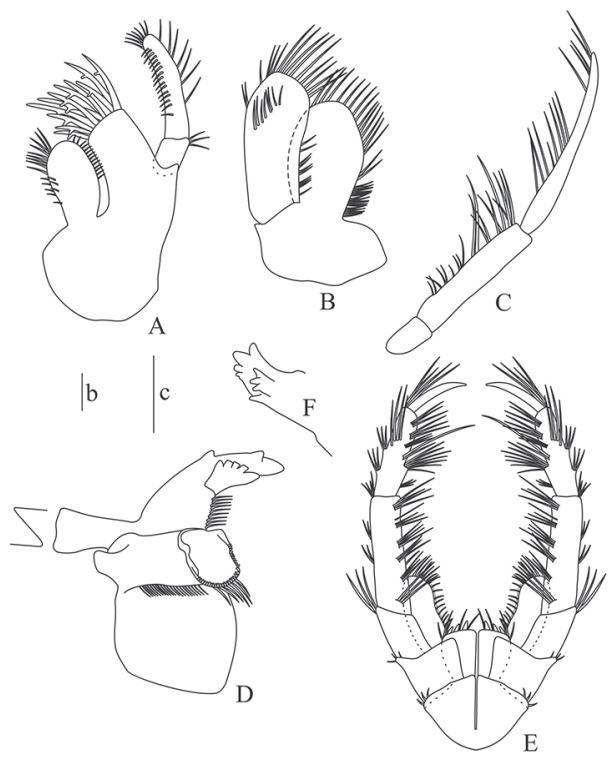

Fig. 2. Amathillopsis antillensis, especie nueva. A. Maxila 1; B. Maxila 2; C. Palpo mandibular; D. Mandíbula derecha; E. Mandíbula izquierda y F. Maxilípedo. Escala. b: $0.2 \mathrm{~mm}$ y $0.6 \mathrm{~mm}$

Fig. 2. Amathillopsis antillensis, new species. A. Maxilla 1; B. Maxilla 2; C. Mandibular palp; D. Right mandible; E. Left mandible and F. Maxilliped. Scale bar. b: $0.2 \mathrm{~mm}$ and $0.6 \mathrm{~mm}$

un proceso espinoso mediodorsal pequeño, pereionitos 5-7 con procesos espinosos mediodorsales largos $\mathrm{y}$ puntiagudos débilmente curvados en su región posterior. Procesos espinosos similares pero un poco más largos sobre los segmentos del pleon 1-2 y uno más corto sobre el pleonito 3. Urosomito 1 tan largo como los urosomitos $2 \mathrm{y}$ 3 combinados, urosomito 3 es el más corto. Urosomito 1 con un proceso espinoso mediodorsal puntiagudo (Fig. 1). Epímero 1 truncado en $\mathrm{su}$ región ventral y redondeado 


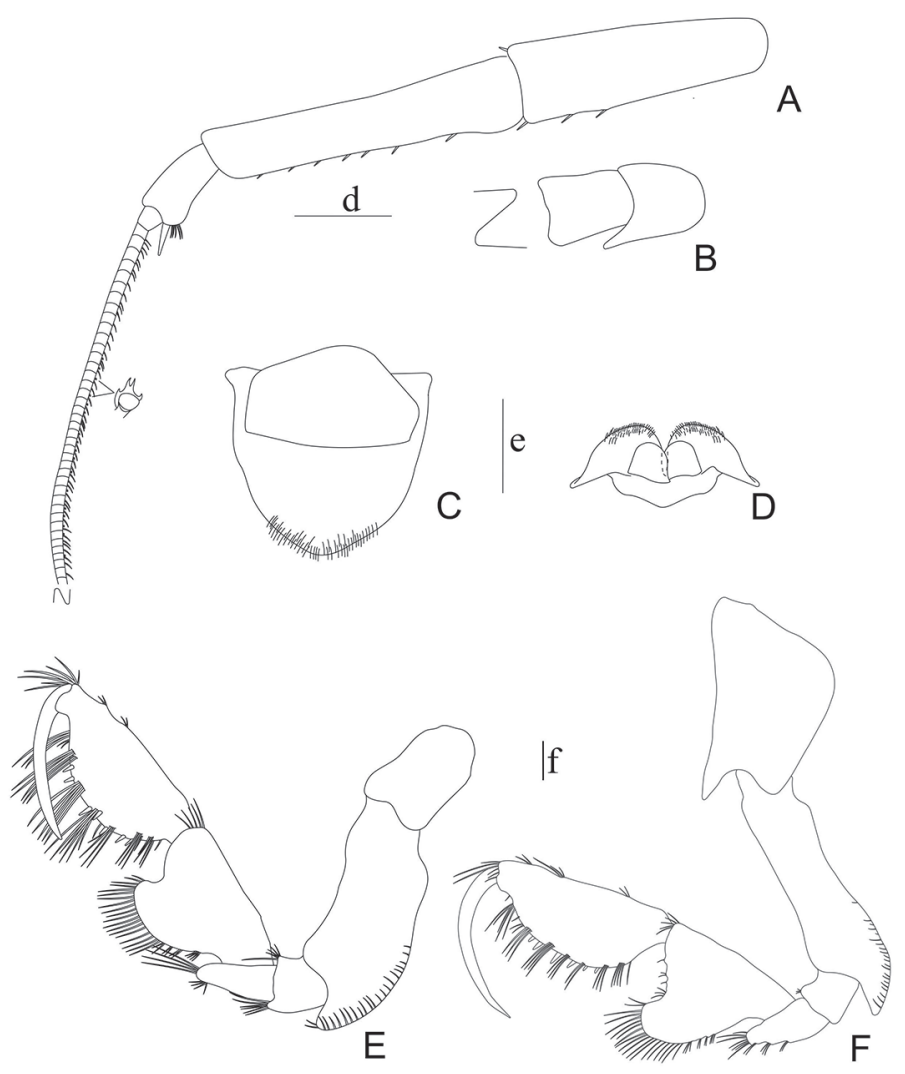

Fig. 3. Amathillopsis antillensis, especie nueva. A. Antena 1 (d); B. Antena 2 (d); C. Labio superior (e); D. Labio inferior (e); E. Gnatópodo 1 (f) y F. Gnatópodo 2 (f). Escala. d: $1 \mathrm{~mm}$; e y f: $0.5 \mathrm{~mm}$

Fig. 3. Amathillopsis antillensis, new species. A. Antenna 1 (d); B. Antenna 2 (d); C. Upper lip (e); D. Lower lip (e); E. Gnatopod 1 (f) and F. Gnatopod 2 (f). Scale bar. d: 1 $\mathrm{mm}$; e y f: $0.5 \mathrm{~mm}$

posteroventralmente; epímero 2 agudo en su región posteroventral; margen posteroventral del epímero 3 sinuoso; ángulo posteroventral agudo (Fig. 5E).

Antena 1, artejo 1 del pedúnculo ligeramente más corto en longitud que el artejo 2; artejo 3 menos de $1 / 3$ de la longitud del artejo 2; flagelo accesorio con solo un artejo, casi dos veces la longitud del artejo 1 del flagelo antenal, flagelo antenal con numerosos artejos. Calceolos presentes (Fig. 3A). Antena 2 dañada, solo permanecen el artejo 1 y el artejo 2, artejo 2 corto es casi la tercera parte de la longitud del artejo 1 de la antena 1 (Fig. 3B). Labio superior casi tan ancho como largo, con margen convexo y entero (Fig. 3C). Labio inferior con lóbulos internos pequeños, lóbulos externos anchos y redondeados distalmente, con grupos de setas finas mediodistales (Fig. 3D). 
Mandíbula derecha, incisivo con seis dientes, lacinia mobilis ancha y con tres dientes puntiagudos; setas auxiliares presentes; molar débilmente surcado con dientes muy pequeños en los márgenes (Fig. 2D). Mandíbula izquierda, incisivo y lacinia mobilis con tres dientes, respectivamente (Fig. 2E). Palpo mandibular con artejos 2 y 3 alargados, con setas largas en sus márgenes ventrales; artejo 3 , delgado y aguzado, la relación entre la longitud del artejo 3 con respecto al artejo 2 es de 1,5 (Fig. 2C). Maxila 1 con lóbulo interno ancho distalmente, con ocho setas plumosas mediodistales; placa externa con 11 setas apicales delgadas; palpo con dos artejos; artejo 1 expandido distalmente; artejo 2 es aguzado con hileras de setas laterales y grupo de setas mediodistales (Fig. 2A). Maxila 2 con la placa externa más larga que la interna; placa interna expandida; ambas placas con setas largas, distales y mesiales (Fig. 2B). Maxilípedo delgado y cubierto con numerosas setas; placas internas cortas con tres setas nodulares medioapicales; placas externas delgadas con hileras de setas mesiales y setas apicales largas; artejos 2-4 del palpo, alargados; artejo 2 es el más largo; artejo 3 con setas largas en su superficie anterior, dáctilo casi tan largo como el artejo 3 (Fig. 2D).

Gnatópodo 1 subquelado, coxa 2/3 de la longitud de las coxas 2-4 y más estrecha que estas, con una excavación anteroventral; margen anterior del basipodito ligeramente recto y margen posterior convexo con setas cortas sub- marginales, que termina en un lóbulo redondeado posterodistalmente. Isquiopodito casi tan corto como ancho con setas largas en el margen distal; meropodito con setas apicales largas; carpopodito expandido distalmente en un lóbulo posterior, cubierto con setas mesial y posteromarginalmente; propodito delgado, más largo que ancho, con el margen posterior convexo con hileras de setas largas en sus caras media y lateral. Dactilopodito delgado y casi de la misma longitud del propodito (Fig. 3E).

Gnatópodo 2 subquelado, con coxa excavada en su región ventral, anteroventralmente aguda; basipodito con margen anterior recto, margen posterior convexo expandido en su región posterior en forma de lóbulo redondeado con setas submarginales cortas, cara mesial con setas largas; isquiopodito más estrecho que en el pereiópodo 1, subcuadrado; meropodito más largo que ancho con grupo de setas apicales; carpopodito expandido distalmente con setas en su cara media y posteromarginal; carpopodito expandido en un lóbulo posterior amplio. Dactilopodito delgado y casi del mismo largo del propodito (Fig. 3F).

Pereiópodos 3-7 dañados, han perdido tres artejos, carpopodito, propodito y dactilopodito, respectivamente. Pereiópodo 3 con la coxa más larga que en los pereiópodos precedentes, ápice oblicuo con una pequeña depresión; basipodito subrectagular; isquiopodito casi tan largo como ancho; meropodito subigual en longitud al basipodito, márgenes ventral $\mathrm{y}$ dorsal con setas robustas pequeñas (Fig. 4A). 


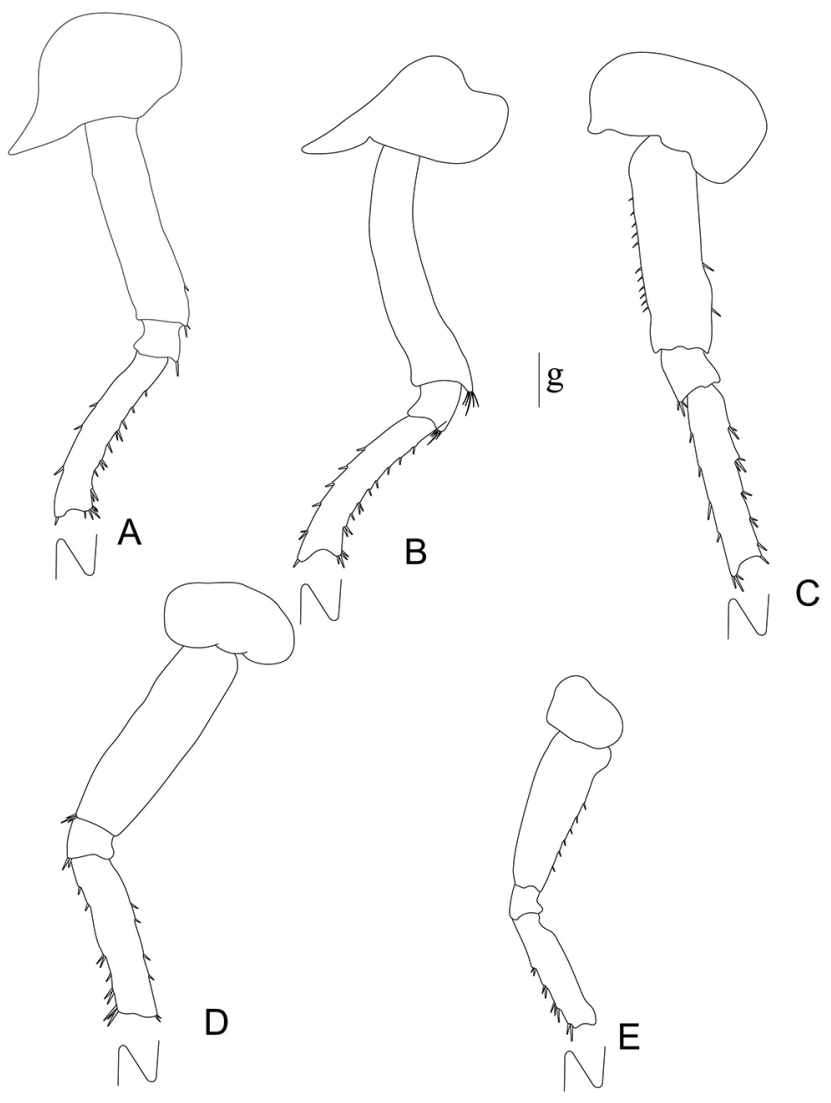

Fig. 4. Amathillopsis antillensis, especie nueva. A. Pereiópodo 3; B. Pereiópodo 4; C. Pereiópodo 5; D. Pereiópodo 6 y E. Pereiópodo 7. Escala. g: $1 \mathrm{~mm}$

Fig. 4. Amathillopsis antillensis, new species. A. Pereiopod 3; B. Pereiopod 4; C. Pereiopod 5; D. Pereiopod 6 and E. Pereiopod 7. Scale bar. g: $1 \mathrm{~mm}$

Pereiópodo 4 con coxa aguzada, subigual en longitud a la del pereiópodo 3; basipodito con márgenes anterior y posterior débilmente convexos; isquiopodito casi tan largo como ancho; meropodito curvado en su región posterior, márgenes ventral y dorsal con setas robustas pequeñas (Fig. 4B). Pereiópodo 5, el más largo de todos, con coxa más ancha que larga; basipodito subrectangular con lóbulo proximal corto y redondeado dirigido dorsalmente; isquiopodito y meropodito excavados en su región posterior, márgenes ventral y dorsal con setas robustas pequeñas (Fig. 4C). Pereiópodo 6 es más corto que el pereiópodo 5 , coxa similar a la del pereiópodo 5 pero algo menor; isquiopodito casi tan largo como ancho; meropodito más corto que el de los pereiópodos 3-5 con márgenes ventral y dorsal con setas robustas pequeñas (Fig. 4D). Pereiópodo 7 es el más corto de todos los pereiópodos, coxa más pequeña que la de los pereiópodos 1-6; basipodito más amplio hacia su región 

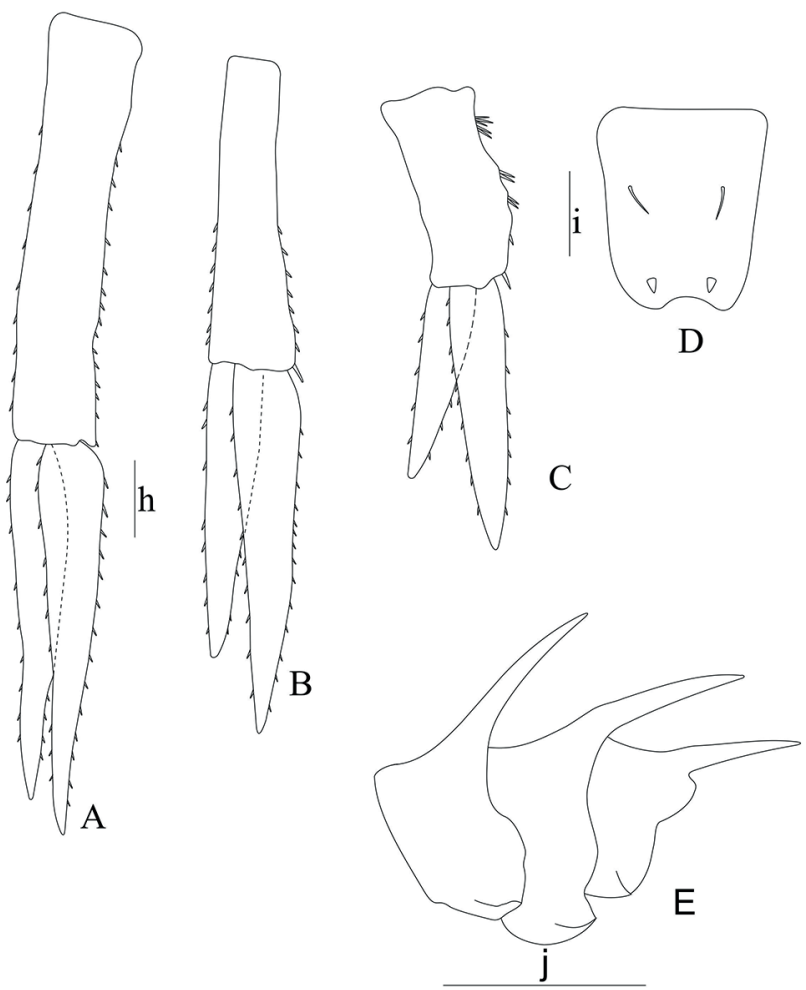

Fig. 5. Amathillopsis antillensis, especie nueva. A. Urópodo 1 (h); B. Urópodo 2 (h); C. Urópodo 3 (h); D. Telson (i) y E. Epímeros de los pleonitos 1-3 (j). Escala. h e i: 0.5 $\mathrm{mm} ; \mathrm{j}: 5 \mathrm{~mm}$

Fig. 5. Amathillopsis antillensis, new species. A. Uropod 1 (h); B. Uropod 2 (h); C. Uropod 3 (h); D. Telson (i) and E. Epimera of pleonites 1-3 (j). Scale bar. h and i: 0.5 $\mathrm{mm} ; \mathrm{j}: 5 \mathrm{~mm}$

proximal, se estrecha ligeramente hacia su porción distal; isquiopodito casi tan largo como ancho; meropodito subrectangular con margen ventral con setas robustas pequeñas (Fig. 4E).

Todos los pedúnculos de los urópodos y ambas ramas uropodales son densamente bordeados con setas espiniformes. Urópodo 1 con el pedúnculo igual a la longitud de la rama interna (Fig. 5A). Urópodo 2 con pedúnculo más corto que ambas ramas, rama externa más corta que la rama interna (Fig. 5B). Urópodo 3 con pedúnculo amplio, con setas robustas en su margen externo, rama externa más corta que la rama interna (Fig. 5C). Telson más largo que ancho, entero, emarginado distalmente, con un par de setas cercanas a su porción media y un par de setas robustas subdistales en la superficie dorsal (Fig. 5D).

\section{DISCUSIÓN}

Amathillopsis antillensis sp. n., carece de ojo, flagelo antenal con 
solo un artejo, gnatópodo 2 con lóbulo ventral del carpopodito amplio y telson emarginado distalmente. Dentro del género solo $A$. atlantica Chevreux, 1908 de las Islas Azores, Groenlandia, el Mar Mediterráneo y Brasil; A. australis Stebbing, 1883 de Australia; A. comorensis Ledoyer, 1986 del Océano Índico y A. pacifica Gurjanova, 1955 del Mar de Okhotsk, comparten estos caracteres.

En Amathillopsis atlantica la relación entre la longitud del artejo 3 del palpo mandibular con respecto al artejo 2 es de 0,8; no presenta proceso espinoso mediodorsal en el pereionito 4 ni en el urosomito 1; mientras que en A. antillensis sp. n., la relación entre la longitud del artejo 3 del palpo mandibular con respecto al artejo 2 es de 1,5; presenta un proceso espinoso mediodorsal en el pereionito $4 \mathrm{y}$ en el urosomito 1 .

En Amathillopsis australis, las coxas 3 y 4 son alargadas; incisivo de la mandíbula izquierda con nueve dientes y lacinia mobilis con seis dientes y no presenta proceso espinoso mediodorsal en el urosomito 1. Sin embargo, en $A$. antillensis sp. n., las coxas 3 y 4 son de tamaño normal; incisivo de la mandíbula izquierda con tres dientes y lacinia mobilis con cinco dientes y presenta un proceso espinoso mediodorsal en el urosomito 1.

En Amathillopsis comorensis, el incisivo de la mandíbula izquierda presenta siete dientes y lacinia mobilis tres dientes y no presenta proceso espinoso mediodorsal en el urosomito 1. Sin embargo, en A. antillensis sp. n., el incisivo de la mandíbula izquierda presenta tres dientes y lacinia mobilis cinco dientes y el urosomito 1 presenta un proceso espinoso mediodorsal.

En Amathillopsis pacifica no se presenta proceso espinoso mediodorsal en el pereionito 4 ni en el urosomito 1 y ambas ramas del urópodo 3 tienen la misma longitud; mientras que $A$. antillensis presenta un proceso espinoso mediodorsal en el pereionito $4 \mathrm{y}$ en el urosomito 1 y la rama externa del urópodo 3 es más corta que la interna.

La presencia del pequeño proceso espinoso mediodorsal en el pereionito 4 parece estar relacionada con el tamaño de los ejemplares. Este solo está presente en el holotipo de 30.2 $\mathrm{mm}$ de longitud total y ausente en los dos paratipos, $20.3 \mathrm{~mm}$ y $28 \mathrm{~mm}$ de longitud total, respectivamente. De esta forma, los paratipos quedarían con procesos espinosos mediodorsales en los pereionitos 5-7, pleonitos 1-3 y urosomito 1. Dentro del género, solo Amathillopsis septemspinosa del Océano Índico, presenta esta misma distribución de procesos espinosos mediodorsales, sin embargo, esta especie se diferencia de $A$. antillensis sp. n., por presentar ojo reducido y el telson no emarginado distalmente.

\section{BIBLIOGRAFÍA}

Barnard, J. L. (1967). Bathyal and abyssal gammaridean Amphipoda of Cedros Trench, Baja California. Bull. $U$. S. Nat. Mus., 260, 1-205. http://dx.doi. org/10.5479/si.03629236.260.1

Barnard, J. L. \& Karaman, G. S. (1991). The families and genera of marine gammaridean Amphipoda (except marine gammaroids). Part 1. Rec. Austr. Mus., 
Suppl. 13(1), 1-417. http://dx.doi.org/1 0.3853/j.0812-7387.13.1991.91

Coleman, Ch. D. \& Coleman Ch. O. (2008). Amathillopsis roroi a new species of Amathillopsidae (Crustacea, Amphipoda) from the Antarctic Ocean. Zoosyst. Evol., 84(2), 143-148. http:// dx.doi.org/10.1002/zoos.200800002

Coleman, C. O. \& Barnard, J. L. (1991). Revision of Iphimediidae and similar families (Amphipoda: Gammaridea). Proc. Biol. Soc. Wash., 104(2), 253-268.

Lowry, J. K. (2006). New families and subfamilies of amphipod crustaceans. Zootaxa, 1254, 1-28.
Tomikawa, K. \& Mawatari, S. F. (2006). A new species of the genus Amathillopsis (Crustacea: Amphipoda: Amathillopsidae) from Japan. Spec. Div., 11, 199-207. Varela, C. \& García-Gómez, J. (2015). Especie nueva de Epimeria (Amphipoda, Epimeriidae) del Golfo de México y el Mar Caribe. Solenodon, 12, 1-8.

Wakabara, Y. \& Serejo, C. S. (1999). Amathillopsidae and Epimeriidae (Crustacea, Amphipoda) from bathyal depths off the Brazilian coast: Brazilian deep water fauna: Results of the oceanographic cruise TAAF MD55. Zoosystema, 21(4), 625-645. 